\title{
Review of Core Power Loss Analysis Using Finite Element Methods
}

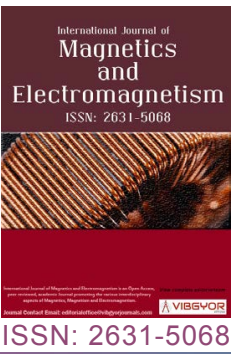

\section{Jorge Rafael González-Teodoro ${ }^{1 *}$, Enrique Romero-Cadaval' and Rafael Asensi ${ }^{2}$}

${ }^{1}$ University of Extremadura, 06006, Badajoz, Spain

${ }^{2}$ Politécnica de Madrid, 28040, Madrid, Spain

\begin{abstract}
There are two principal methods are utilized to estimate the core power loss (CPL) in magnetic components: Empirical equations and hysteresis models. Both approaches need an anterior test or analysis to calculate the CPL. Most of them are based on improved Steinmetz equations. Tests are usually costly or the number of necessary tests is excessive for the component design development. 2D analyses using Finite Element are not good enough for several magnetic components for their lack of symmetries.

Non-symmetric magnetic cores are ones of the most common components in transformers and power converters and they don't have symmetries on the magnetic field distribution and only 3D models are competent to take account all the effects and phenomena.
\end{abstract}

\section{Keywords}

Core power loss, Eddy current loss, Hysteresis loss, Finite element analysis

\section{Introduction}

Losses in magnetic cores have been analyzed because of their particular importance to the component design in power electronics. Physicists study the features in magnetic materials, while design engineers in power electronics need to model the CPL. Nevertheless, there is a break between the power loss calculation theories, focused in material characteristics, and engineering applications. In consequence the devices designed cannot fully use the material capabilities.

One group of models are based on the Steinmetz equation where the coefficients are determined by fitting the loss model to the measurement experimental data. This model postulates purely sinusoidal flux densities. An increase of [1] was presented by Jordan in [2] where an iron loss model where the static hysteresis loss ( $\mathrm{HL}$ ) and dynamic eddy current loss (ECL) were divided. Pry and Bean added the excess loss factor or anomalous factor [3]. Bertotti developed a theory developing the losses by introducing the concept of magnetic objects, which led to excess loss in terms of the active magnetic objects and the domain wall motion $[4,5]$. Once the separation of the iron losses after the magnetizing processes was added, the losses caused by linear magnetization, rotational magnetization and higher harmonics were involved $[6,7]$.

In the last times, the Steinmetz equation was im-

*Corresponding author: Jorge Rafael González-Teodoro, University of Extremadura, 06006, Badajoz, Spain

Accepted: October 14, 2019; Published: October 16, 2019

Copyright: (c) 2019 González-Teodoro JR, et al. This is an open-access article distributed under the terms of the Creative Commons Attribution License, which permits unrestricted use, distribution, and reproduction in any medium, provided the original author and source are credited.

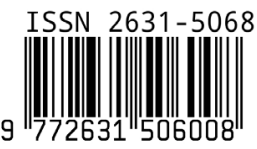

González-Teodoro et al. Int J Magnetics Electromagnetism 2019, 5:023 
proving. The Modified Steinmetz equation was presented in [8] for arbitrary waveforms and the Generalized Steinmetz equation [9] that presented the idea of the instantaneous iron loss is a single valued function of the flux density and the rate of change of the flux density. The improved Generalized Steinmetz equation [10] was developed to avoid the limitation in the third higher harmonic. For rectangular shapes voltages, the improved-improved generalized equation was published in [11].

To obtain a higher accuracy, hysteresis models (Preisach and Jiles-Atherton) were developed [12,13]. An improvement of the Preisach model was presented in [14] including a dynamic part divided in two sections describing the low and high values of the flux density. A friction hysteresis model based on energy approach where the magnetic dissipation from the macroscopic point of view is represented by a friction-like force was introduced in [15]. Advances for nonlinear behaviour were presented in [16]. A new model which describes the switching behaviour implemented in Matlab is in [17]. Analysis for non-sinusoidal signals for specific components used currently in power electronics has been studied in the last years [18-25].

The empirical and separation loss models are preferable and best suited for fast and rough iron loss definition. The complex hysteresis loss models are more adequate for an exact iron loss. They need much more knowledge about the material data or prior material measurements as well as more information about the flux density waveforms. Another huge issue is the integration into Finite Element tools.

\section{Physics Analysis}

The core power losses are based on Joule heating [26] and due to spin relaxation, although this last impact is only at frequencies in the medium-high frequencies. Hysteresis and eddy current losses are caused by the same physical phenomena: Every change in magnetization (which also occurs at DC magnetization) is a movement of domain walls and produces eddy currents creating Joule heating. The fact that hysteresis losses also arise at almost zero frequency is due to the macroscopic magnetization change, even it is slowly, the local magnetization the domains modifies quickly, which generates eddy current losses. In consequence, the engineering approach of loss separation into different loss types (the so-called hysteresis losses, eddy current losses and excess losses) is an empirical approach, trying to separate the different physical influences due to frequency and flux density variations in electromagnetic systems, rather than explaining the physical phenomena directly [27].

\section{Engineering Analysis}

The referenced break is produced because engineers usually design devices based on models with different coefficients than physicists use on their models.

However, these models are normally appropriate only for a defined frequency range, and practical disadvantages of most of these methods require additional measurements with a given material. Another additional issue is that these methods are only for static situations [1]. Other limitations are:

1) The models are based on eddy loss induced by domain wall motion; it doesn't match with empirical data for ferrites (Steinmetz equation coefficients).

2) It needs material and magnetic field data. Thus, it requires a simulation or essay to estimate the magnetic field into the core.

\section{Core Power Loss Analysis using FEM}

Most of the FEM $[28,29]$ uses the Steinmetz equation with the defined parameters from the software or coefficient adjustments from the manufactures sheets on the program settings.

Also, there is another method to calculate the core power loss using FEM defined in [28] for the hysteresis and eddy current losses.

Hysteresis loss is flowing in the forward and reverse directions magnetizes and demagnetizes the core alternatively during each AC cycle. This energy loss is dependent of the properties of particular core material and proportional to the hysteresis loop area.

$$
P_{\text {hysteresis }}=\int_{\text {vol }} \frac{1}{2} \cdot \omega \cdot \operatorname{Im}(\vec{B} \cdot \vec{H} \cdot) d V
$$

Where $\vec{B}$ is the magnetic flux density, $\vec{H}$ is the complex conjugate of the magnetic field and $\omega$ is the angular frequency.

Eddy current loss is an electric current step up by an alternating magnetic field. These losses arise from the fact that the core itself is composed of conducting material, so that the voltage induced in it by the varying flux produces circulating currents in the material. Eddy current loss depends upon the rate of change of flux as well as the resistance of the path, it is reasonable to expect this loss varies as the square of both the maximum flux density and frequency if the core is solid and made up from ferromagnetic materials, and it effectively acts as a single short circuited turn. Induced eddy currents therefore circulate within the core in a plane normal to the flux, and cause resistive heating of the core material. 


$$
P_{e d d y 1}=\int_{v o l} \frac{1}{2 \sigma} \cdot(\vec{J} \cdot \vec{J}) d V
$$

Where $\vec{J}$ is the current density, $\vec{J}$ is the complex conjugate of the current density and $\sigma$ is the conductivity in a structure.

For the component surfaces, the eddy current loss is defined by:

$$
P_{e d d y 2}=\sqrt{\frac{\omega \mu_{o} \mu_{r}}{8 \sigma}} \cdot \int \vec{H}_{t} \cdot \vec{H}_{t} d s
$$

Where $\vec{H}_{t}$ is the tangential component of the $\vec{H}$ on the boundary and $\vec{H}_{t}$ is the complex conjugate tangential component of $\vec{H}$ on the boundary.

Nevertheless, the modeling in FEA depends on the core symmetry and winding configuration. This dependency produces that the loss calculation in non-symmetric components through a 3D FEM is a difficult task due to the current hardware computational limitations [30].

The current computational limitations [31,32] provoke to perform some simplifications at the component modeling to achieve the performance of the 3D FEM simulation in operative way for the design engineers. The allowed simplifications depend on the magnetic field distribution (1D/2D/3D).

2D Models are not enough precise in FEM when there is non-axial symmetry. In addition, there important effect that there are only possible to analyze in 3D models as the air-gap and edge effects.

The air-gap effect defines when in the magnetic core is introduced a material with poor permeability (usually it's air), there is a discontinuity in the core. This situation produces that the magnetic flux disperses in the closed zone to the air-gap. The disperse flux affects the current distribution of the conductors near of the air-gap. This phenomenon is very similar to the skin and proximity effect. The current of the conductors tries to minimize the energy with its own distribution, so, the real section decreases and the losses increase.

The edge effect consists in the direction change of the flux when the conductors finish. In each winding belongs to the magnetic component, the conductors fill the high of the window, and then, the distribution of the field is practically in the same direction, so, when the field arrives at the up or down of the core window, the field has to change the direction and it appears another new distribution different the main one.

Also, when it is winded the primary and secondary in the same split of the component, this effect appears in the edge and in the space of the two closed turns of the winding modifying the magnetic field density into the core.

For power engineers, the equivalent parameters ( $L$ and $\mathrm{R}$ for the core) can be interesting for the optimization of the component.

The estimation $L$ is by 3D simulation and the $L-/$ curve described in [33] can be define using one of the outputs ( $\phi$-I curve) by differentiations as:

$$
L(I)=\frac{d \phi}{d l}
$$

Where $\phi$ is the magnetic flux.

To obtain $R$, it is derived at the post-modeling step $\mathrm{R}-I_{r m s}$ curve by numerical integration described in [33] as:

$$
R=\frac{\bar{p}}{l_{r m s}^{2}}
$$

Where $\bar{p}$ the average value of the power and $I_{r m s}$ is the $r m s$ value of the current on the windings.

\section{Conclusion}

As it is mentioned previously, the complex hysteresis loss models are more adequate for an exact iron loss, however there is a huge issue is the integration into $\mathrm{Fi}$ nite Element tools.

The hardware computer limitation is not useful in CPU time spending for the power electronics engineers and the 3D FEM in higher frequencies (> $10 \mathrm{MHz}$ ), the time consuming is especially long and the error increases substantially.

There are different ways to get along with these limitations. Some simplifications in the 3D model could be a solution, but these kind of limitations may become in the next bottleneck for future works.

Another choice is moving the research to decrease the Finite Element numbers reducing the space moving closer the boundaries using the perfectly matching layers technique.

\section{References}

1. Ch P Steinmetz (1984) On the law of hysteresis. Proc IEEE 72: 196-221.

2. H Jordan (1924) Die ferromagnetischen Konstanten für schwache Wechselfelder. Elktr Nach Techn 1.

3. RH Pry, CP Bean (1958) Calculation of the energy loss in magnetic sheet materials using a domain model. Journal of Applied Physics 29: 532-533.

4. G Bertotti (1958) Physical interpretation of eddy cur- 
rent losses in ferromagnetic materials. II. Analysis of experimental results. Journal of Applied Physics 57: 2118-2126.

5. G Bertotti (1988) General properties of power losses in soft ferromagnetic materials. IEEE Transactions on Magnetics 24: 621-630.

6. T Kochmann (1996) Relation between rotational and alternating losses in electrical steel sheets. Journal of Magnetism and Magnetic Materials 160: 145-146.

7. L Michalowski, J Scheinder (2006) Magnettechnik-Grundlagen, Weksoffe, Anwendugen. ( $3^{\text {rd }}$ edn), Vulkan-Verlag GmbH.

8. J Reinert, A Brockmeyer, RWAA De Doncker (2001) Calculation of losses in ferro and ferromagnetic materials based on the modified Steinmetz equation. IEEE Transactions on Industrial Applications 37: 1055-1061.

9. J Li, T Abdallah, CR Sullivan (2001) Improved calculation of core loss with no sinusoidal waveforms. In: Industry Applications Conference, $36^{\text {th }}$ IAS Annual Meeting, conference Records, 4: 2203-2210.

10.K Venkatachalam, CR Sullivan, T Abdallah, H Tacca (2002) Accurate prediction of ferrite core loss with nonsinusoidal waveforms using only Steinmetz parameters. IEEE workshop on computers in power electronics 36-41.

11.J Muhlethaler, J Biela, JW Kolar, A Ecklebe (2012) Improved core-loss calculation for magnetic components employed in power electronic systems. IEEE Transactions on Power Electronics 27: 964-973.

12.F Preisach (1935) Über die magnetische Nachwirkung. Zeitschrift für Physik 94: 277-302.

13.D Jiles, D Atherton (1986) Theory of ferromagnetic hysteresis. Journal of Magnetism and Magnetic Materials 61: 48-60.

14.(2007) Flux 10-2D/3D Applications User's guide. Cetrat Group.

15.M Hafner, F Henrotte, MH Gracia, K Hameyer (2008) An energy-based harmonic constitutive law for magnetic cores with hysteresis. IEEE Transactions on Magnetics 44: 922-925.

16.D Eggers, S Steentjes, K Hameyer (2012) Advanced iron-loss estimation for nonlinear material behavior. In IEEE Transactions on Magnetics 48: 3021-3024.

17.D Linzen, RW De Doncker (2002) Simulation of power losses with MATLAB/Simulink using advanced power device models. IEEE Workshop on Computers in Power Electronics, Proceedings, Mayaguez, Puerto Rico, USA, 71-75.
18.Sobhi Barg, Kaiçar Ammous, Hanen Mejbri, Anis Ammous (2017) An improved empirical formulation for magnetic core losses estimation under nonsinusoidal induction. IEEE Transactions on Power Electronics 32 .

19.Shuaichao Yue, Yongjian Li, Qingxin Yang, Xinran Yu, Changgeng Zhang (2018) Comparative analysis of core loss calculation methods for magnetic materials under non-sinusoidal excitations. IEEE Transactions on Magnetics 54.

20.Shuaichao Yue, Qingxin Yang, Yongjian Li, Changgeng Zhang, Guizhi Xu (2017) Core loss calculation of the soft ferrite cores in high frequency transformer under non-sinusoidal excitation. $20^{\text {th }}$ International Conference on Electrical Machines and Systems (ICEMS), Sydney, NSW, Australia.

21.Satoshi Shimokawa, Hirotaka Oshima, Koichi Shimizu, Yuji Uehara, Jun Fujisaki, et al. (2018) Fast 3-D optimization of magnetic cores for loss and volume reduction. IEEE Transactions on Magnetics 54.

22.Georg Von Pfingsten, Simon Steentjes, Kay Hameyer (2017) Operating point resolved loss calculation approach in saturated induction machines. IEEE Transactions on Industrial Electronics 64.

23.Qiang Yu, Berker Bilgin, Ali Emadi (2015) Loss and efficiency analysis of switched reluctance machines using a new calculation method. IEEE Transactions on Industrial Electronics 62.

24. Arash Khoshkbar Sadigh, Vahid Dargahi, Keith A Corzine (2016) Investigation of conduction and switching power losses in modified stacked multicell converters. IEEE Transactions on Industrial Electronics 63.

25.Limin Yang, Yaohua Li, Zixin Li, Ping Wang, Shukai Xu, et al. (2019) A simplified analytical calculation model of average power loss for modular multilevel converter. IEEE Transactions on Industrial Electronics 66.

26.CD Graham (1982) Physical origin of losses in conducting ferromagnetic materials. Journal of Applied Physics 53: 8276-8280.

27.Andreas Krings, Juliette Soulard (2010) Overview and comparison of iron loss models for electrical machines. EVER Monaco.

28.https://www.ansys.com/Products/Electronics/ANSYS-Maxwell

29.https://www.ansys.com/en-in/products/electronics/ansys-pexprt

30.Benoît Delinchant, Frédéric Wurtz, Joao Vasconcelos, Jean-Louis Coulomb (2014) Framework for the optimization of online computable models. COMPEL 
International Journal of Computations and Mathematics in Electrical 33: 745-758.

31.llona latchev (2018) Application of field modeling and determination of parameters in electrical engineering education. COMPEL International Journal for Computation and Mathematics in Electrical and Electronic Engineering 37: 1585-1596.
32.Dmitry S Kiselev, Marina G Persova, Yuri G Soloveichik (2016) Actual problems of electronics instrument engineering (APEIE). $13^{\text {th }}$ International Scientific-Technical Conference, Novosibirsk, Russia.

33.RA Salas, J Pleite (2013) Equivalent electrical model of a ferrite core inductor excited by a square waveform saturation and power losses for circuit simulation. IEEE Trans Magnetics 49. 